\title{
Design and Implementation of Medical Wireless Nursing Call System
}

\author{
Juan Wan, Ya Wang \\ School of Intelligence Science and Information Engineering \\ Xi'an Peihua University, Xi'an, China \\ 312610191@qq.com,1042407619@qq.com
}

\section{Keywords: Radio transmission and reception; Wireless transmission system}

\begin{abstract}
The appeal information of patients is very important, and each piece of information is related to a living life. Calling system becomes a bridge between the doctor and the patient. Compared with the traditional wired calling equipment, the wireless transmission system design and implementation will save a lot of wiring funds for the hospital.This project is a wireless calling system, which adopts a wireless codec transmission method, overcomes the problems of troublesome wiring and reconstruction of the wired calling system under the traditional situation. The wireless calling system designed this time is mainly divided into two major parts: transmit and receive. The system includes a plurality of call remote control, which can meet the needs of multiple beds or wards. With the data codec chip PT2262 and PT2272 as the core, as well as the wireless transceiver module used for transmission. The number of the caller is displayed on the nurse station LED digital display screen with an audible prompt.
\end{abstract}

\section{Introduction}

With the rapid development of wireless communication technologies, more and more people are beginning to recognize and use this technology. In the past, a mature system in wireless communication - cellular system in many countries has become an indispensable communication tool for work and daily life. Many homes, business districts and campuses have already opened wireless LANs. It is supplementing or even replacing wired networks.

With the continuous deepening of the reform of the medical system, people increasingly need to quickly and easily obtain various medical services in the hospital. The quality of service and the speed of response have gradually become the focus of a comprehensive hospital's medical care. Along with this, a hospital wireless calling system came into being.

\section{System Overview}

\subsection{Overall System Design}

When designing a wireless calling system, the system's scheme is firstly selected according to the characteristics of the wireless calling and the requirements for system performance, mainly considering the wireless module and the one-chip computer connection and the influence of circuit on signal of receiving and transmitting module. The wireless simulation calling system has the following scheme:

Using PT2272 and PT2262 transceiver chip, the system works in the vicinity of $315 \mathrm{Mhz}$. This program uses a modular design, its transmission and reception are based on PT2262 and PT2272 chip-based integrated module, so there is no disturbing phenomenon in scheme 1 . The program is 
mainly a modification of scheme 1 , the integrated module is cheap, economical and practical. The overall block diagram of the system is shown in Fig.1.

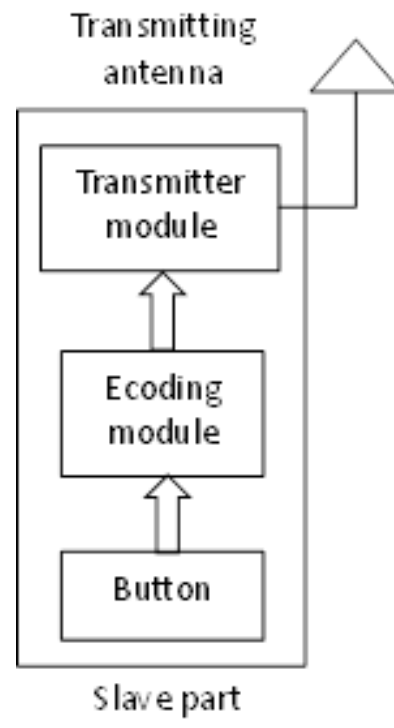

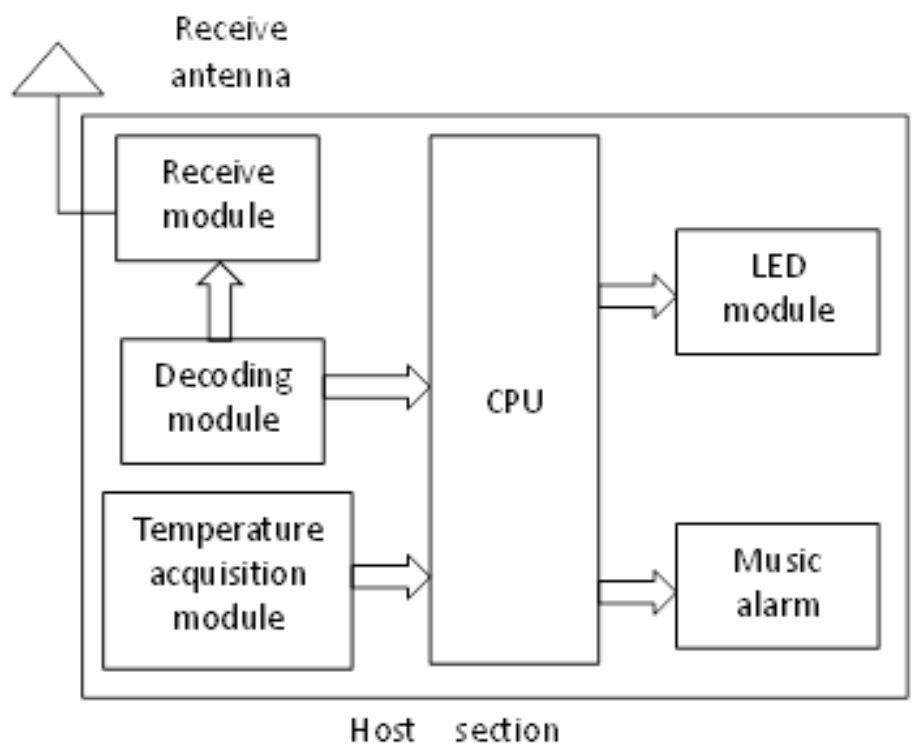

Figure 1. Block diagram of ward calling system

\section{2 design of transmitting and receiving modules}

The transmitter is composed of an electromechanical circuit, an oscillator circuit, a ASK circuit, a high frequency power amplifier and an antenna. Oscillating circuit can be regarded as a converter that converts DC power to AC power supply. The carrier generator in the following figure has such functions, and is the basic unit of the wireless transmission circuit. ASK modulation is the application of the digital baseband signal to be applied to the loading process of high-frequency current. The design of high frequency power amplifier is to increase output power, and enable the antenna to radiate through antenna when it reaches the transmit power requirement.

The receiving circuit mainly includes antenna, high frequency small signal amplifier and ASK demodulation circuit. The receiving station converts the received electromagnetic wave to the modulated wave current through the antenna, and then detects the original transmitted signal from the modulation wave current. Because the electromagnetic wave received by the antenna is very weak, it is necessary to increase the small signal amplifier from the first to the next level before demodulation, and then execute it again. After demodulation is completed, the original signal can be restored to a proper low frequency amplifier circuit.

\subsection{Design Principles of Encoding and Decoding Modules}

In the daily development process, 8-bit address code and 4-bit data code are often used. In this case, pin 1 to pin 8 of the encoding circuit PT2262 and decoding PT2272 are address setting pins, which can be suspended, connected to the power supply, and grounded. Only the address codes of the transmitting terminal PT2262 and the receiving terminal PT2272 can be paired and used. If only the PT2262 and PT2272 pins 1 to 8 are set the same, the user can change the address code; when the two addresses are completely the same, the D1 to D4 terminal corresponding to the receiver export 4V interlocking high level control signal, and the VT terminal also outputs the effective high level signal. The user can increase these signals by one level and can drive relays, power transistors, etc. to carry out remote control of the load switch.

\subsection{CPU Program Selection}

Due to the limited resources required for the system, the requirements for the control chip are not very high, and there is no need for very powerful functional deployment. Compared with the 
commonly used main control chip, the SCM has a higher resource utilization rate, and the market price of the SCM is at the middle and lower price level. Since a variety of data can support the SCM's advantages, finally, the main control chip used in the design of the system is AT89S52.

Because AT89S52 chip is not only a low-power, high-performance CMOS 8-bit microcontroller, but also fully compatible with the MCS-51 microcontroller product instructions and pins, with 8k in-system programmable Flash memory, it allows program memory to be programmed in the system.

\section{Hardware Design}

\subsection{Microcontroller Minimum System}

The minimum system of the single chip computer mainly includes AT89S52 single chip computer, clock circuit and reset circuit and other important circuits. The clock circuit is not designed separately and uses an internal clock. Using a high-gain inverting amplifier inside the microcontroller, a self-oscillating circuit consisting of a crystal and a capacitor is connected between XTAL1 and XTAL2 so that the pulse from the oscillator can be fed into the internal clock circuit. This circuit design has two functions: power-on reset and manual reset. The button is the reset button. When the minimum system of the SCM is powered on, the R1 and C1 charge and discharge principles are used to externally supply the RST pin with a high level over 2 machine cycles to achieve the reset of the minimum system of the SCM. The schematic diagram is shown in Fig.2.

\subsection{Codec Circuit Design}

\section{(1)Coding Circuit Design}

The address is grounded, that is, all set to 0. Binary data is input through the data input terminal. The data pins are externally set by the jumper pins, and then the jumper cap is used to connect to the $+5 \mathrm{~V}$ power supply or to ground the address code and data code and export to the data input terminal of high-frequency circuit by data output terminal. The encoding principle is shown in Fig.3.

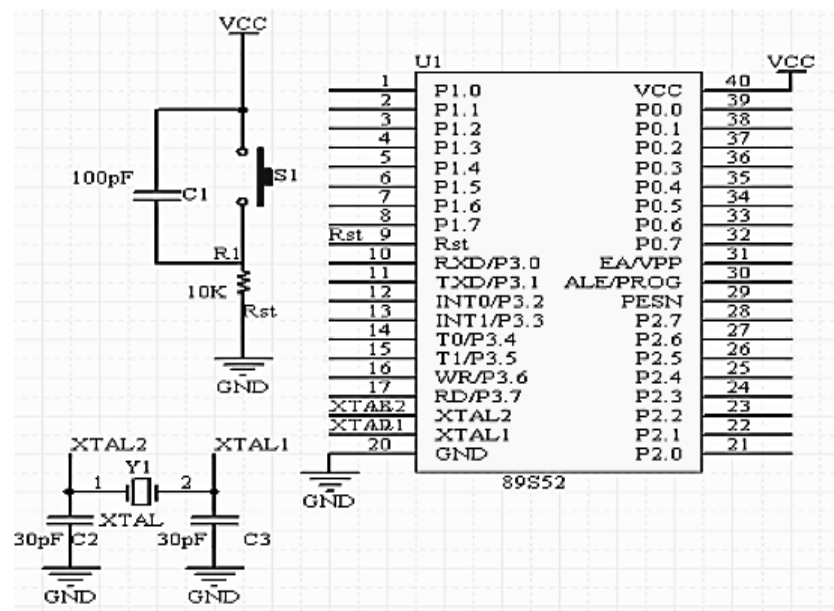

Figure 2. Microcontroller minimum system

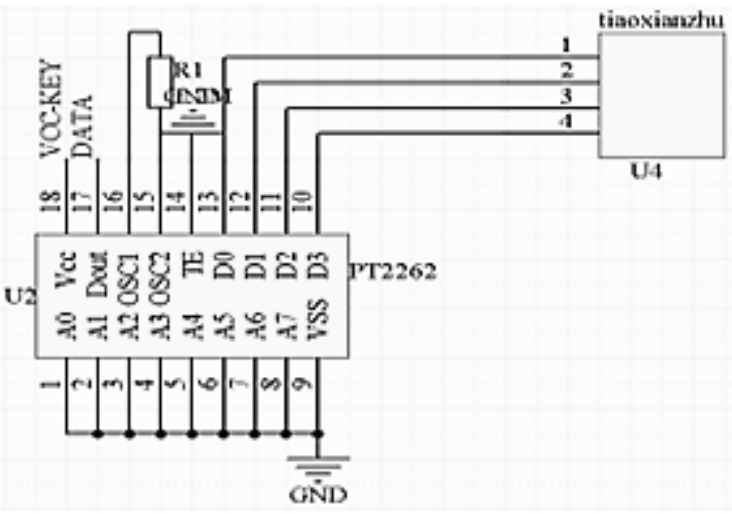

Figure 3. Coded circuit diagram

(2)Decoding Circuit Design

The address code must be set to be the same as the coded address code which is the premise of decoding so that the address port is be fully grounded. The microcontroller can obtain the effective information whether the decoded information is required by the VT port and data received by a super heterodyne receiving circuit input from the DIN end will be sent to the PT2272 for decoding. 
The decoded data will still be sent to the SCM through the data output terminal for information processing. The schematic of the decoder circuit is shown in Fig.4.

\subsection{LCD Display Circuit}

The liquid crystal module is connected to the IO port of the single-chip microcomputer through the exclusion connection method, so as to realize the data transmission. If $\mathrm{R} / \mathrm{W}$ is grounded, the command or data will be written to the LCD module. When RS is connected to P2.6 of the SCM, it will control whether it is a write command or data. Connecting VO to a sliding rheostat, the function of adjusting the brightness of the LCD display can be achieved. If you need to display related information through the LCD, the interface circuit between it and the microcontroller is shown in Fig.5.

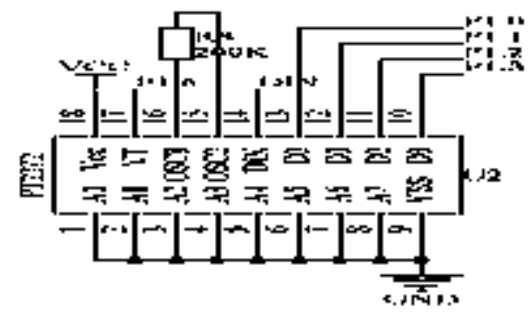

Figure 4. Decoding circuit diagram

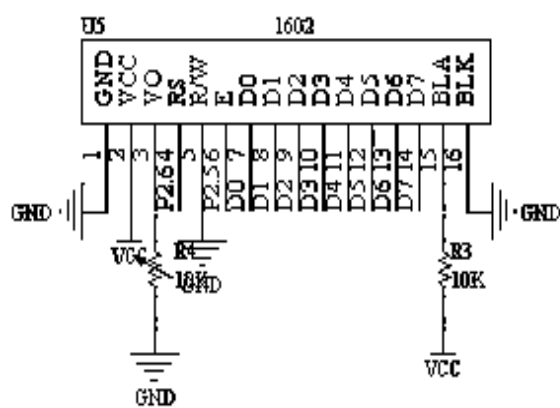

Figure 5. LCD display circuit

\subsection{Music Alarm Circuit}

The music alarm circuit uses the doorbell chip TQ33A which is a common one with 16 songs on the market. The advantage of this chip is that it contains a built-in audio driving circuit that can directly connect speakers to play beautiful music. It is possible to switch the control songs by pressing the button S2, and to control whether the music needs to be played through the P3.2 pin control of the single chip microcomputer. The generated sound needs to be sent through an external speaker. The circuit diagram is shown in Fig.6.

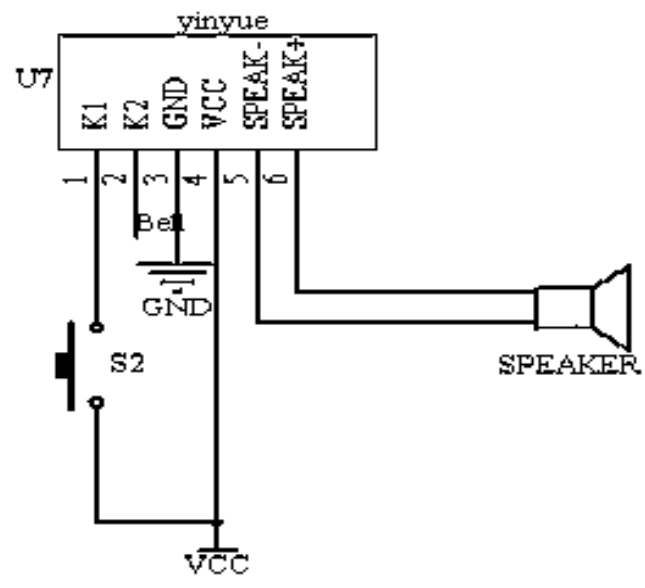

Figure 6. Music alarm circuit

\section{Software Design}

A good combination of hardware and software will make the system work normally. The hardware part determines the basic structure of the system, but the software is the soul of the driving system. The two are interdependent and inseparable. After the host is on, performing various initialization operations on the chip and the device, it enters a data waiting acceptance mode. If a slave signal is received, it will be pre-processed so that the information is displayed on the liquid crystal display, and control music alarms for the purpose of alerting medical personnel. The 
design includes a main program, an interrupt subroutine, a wireless transceiver program, a liquid crystal display program, an alarm music switching subroutine, and so on.

\subsection{Program Flow Chart}

The boot screen is displayed first, and it can be judged whether there is a call in the current state. If the unattended call is in a silent state, the screen displays the current temperature and the displayed common information like "unanswered". When there is a call, the screen will display specific information such as the number of calls and the bed number of corresponding caller. At this time, the music alarm will be activated. When the switch key is pressed, the external interrupt will be activated, and the song name will be changed. After the medical staff who handles the call event press the message clear button, the call information on the LCD will be cleared and reset, indicating only the display temperature and the unattended call. The flow chart is shown in Fig.7.

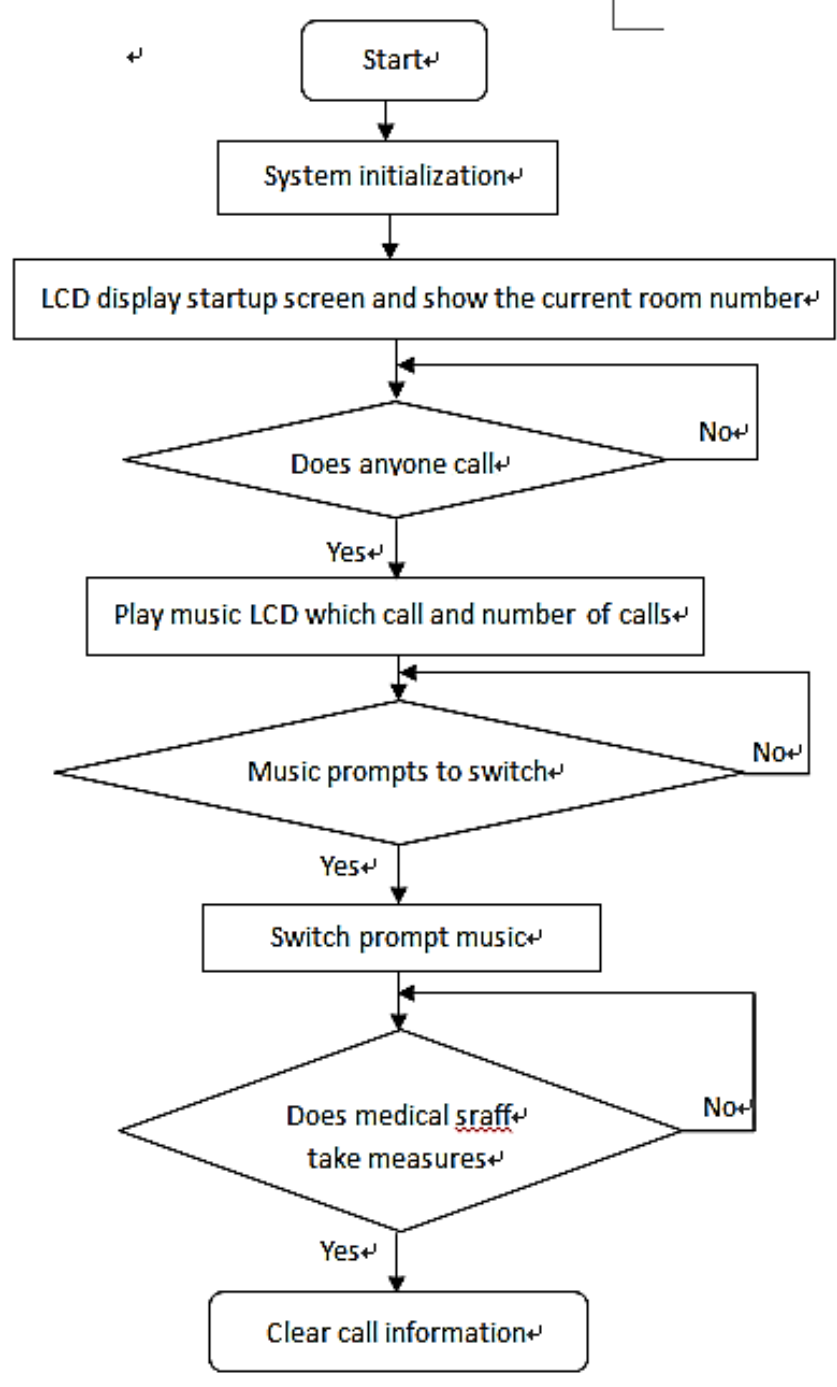

Figure 7. Single-chip microcomputer main program flow chart

\subsection{Liquid Crystal Display Subroutine}

It is used for LCD display for displaying real-time temperature and call information. After the startup screen, it enters into a stable working state, including the clearance of the contrast temperature changes and calling information changes. The flow chart is shown in Fig.8. 


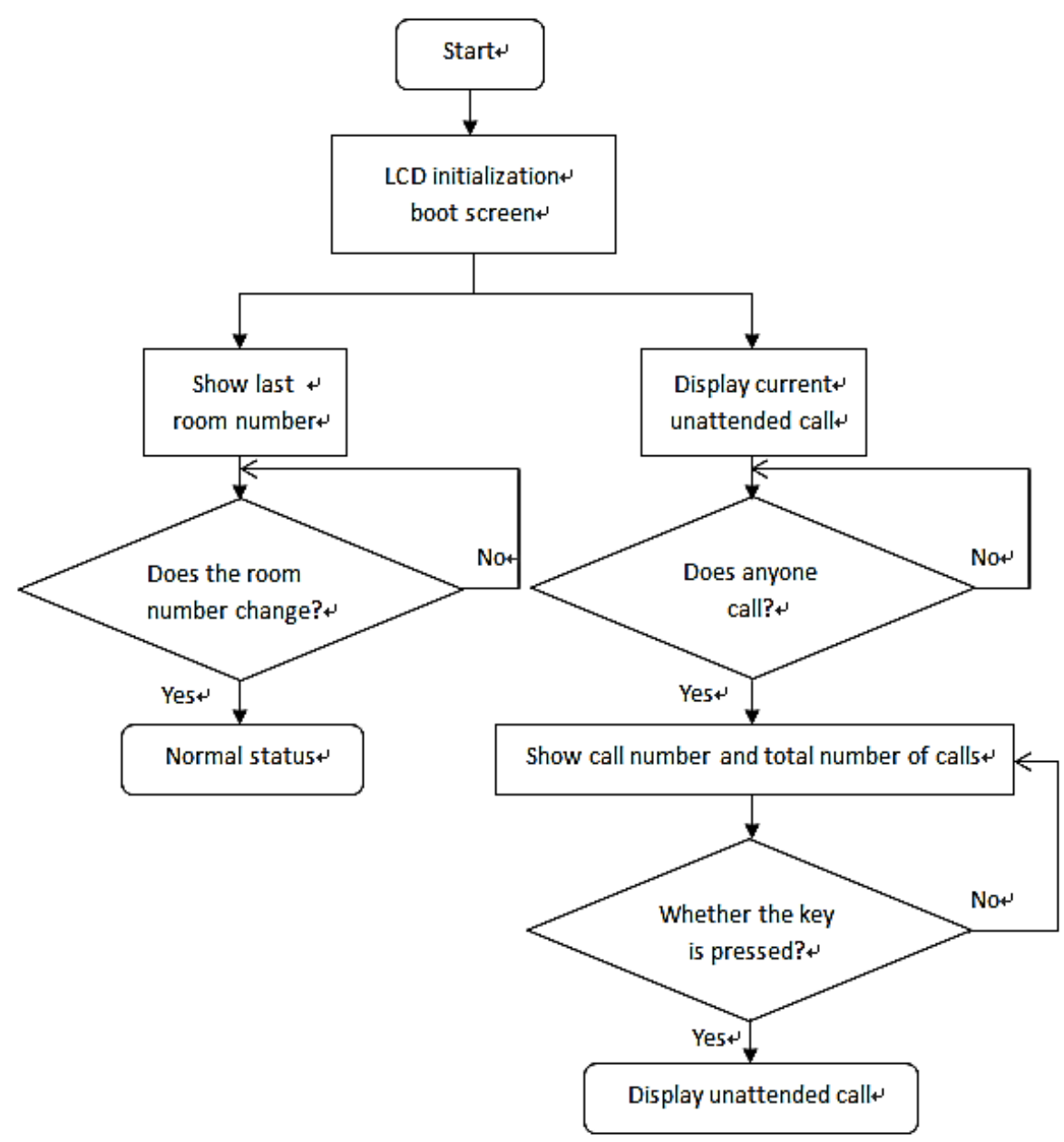

Figure 8. LCD display flow chart

\section{Conclusion}

This system is not only suitable for hospital wards, but it can also be widely applied to anti-theft systems that require multiple precautions, management of large parking lots, hotel room calls to the general station, Q\&A in teaching, wireless model aircraft, wireless remote control toys with multiple actions and other occasions if it is improved.

\section{References}

[1] Chaoqing Li, Jin Lu, Zhiyong Wang, Qiping Yuan. 2017. The principle and interface technology of single chip microcomputer[M]. Beijing university of aeronautics and astronautics press.

[2] Radio editorial department. 2014. $30 \mathrm{MCU}$ stunning display driver program[M]. People's post and Telecommunications Press.

[3] Yang Xu, Zhongwen Tang, Xun Zhang. 2009. Analysis of medical wireless call system[J]. Gansu science and technology.

[4] Longfei Huang, Xiaofeng Huang. 2016. Design of wireless medical call system based on ZigBee[J]. Digital technology and Application. 